\title{
Particle Physics Simulator for Scientific Education using Augmented Reality
}

\author{
Hasnain Hyder ${ }^{1}$, Gulsher Baloch ${ }^{2}$, Khawaja Saad ${ }^{3}$, Nehal Shaikh ${ }^{4}$, Abdul Baseer Buriro ${ }^{5}$, Junaid Bhatti ${ }^{6}$ \\ Department of Electrical Engineering \\ Sukkur IBA university
}

\begin{abstract}
In this era of fourth industrial revolution, young learners need to be equipped with $21^{\text {st }}$ century skills, such as critical thinking, creativity, communication, collaboration, innovation and problem solving. Augmented Reality (AR) based learning systems are an effective tool to embed these skills. This paper presents a detailed review of latest research on an AR-based learning systems. Furthermore, an AR-based learning system is proposed to demonstrate the particle physics experiments i.e. proton-proton collision and Higgs field. The proposed learning system algorithms are developed using particle system of unity 3D software. Then, Microsoft Kinect sensor is interfaced with unity 3D to create an immersive experience. Then, the qualitative analysis of the proposed system and latest AR-based learning systems is presented. Finally, the quantitative analysis of the proposed system is conducted. Overall, the results suggest that $85 \%$ of the participants recommended the proposed learning system.
\end{abstract}

Keywords-Particle physics; augmented reality; proton-proton collision; Higgs field; interactive classroom; AR in education; $A R$ based lab experiments

\section{INTRODUCTION}

According to European Union, AR will be one of the emerging technologies to pave the way for the development of smart industry in near future [1], [2]. The disruptive technologies are extensively utilized in many applications to enhance their performance. However, many sectors are still lacking in adapting the latest technology. Education sector is considered as one of them [3]. In the traditional form of learning, the teacher delivers knowledge while students act as recipients only. However, students find interactive way of learning to be more exciting and effective. AR have a key role in developing learning systems to make the learning process more effective and less tedious [4]. It enables human-machine interaction while overlaying virtual components on real world environment. It has potential applications in multiple fields such as education, health-care, rehabilitation, etc. [5]-[7]. It is combined with holographic technology to create applications for museums and other visiting places to display art and culture [8], [9]. It helps to create new and more effective learning systems to develop critical thinking, creativity, communication, collaboration, innovation and problem solving.

The concept of AR was introduced in the early 90s. Since then, significant advancements have been made in this field [10]. These advancements have created multiple opportunities to develop systems and products that provide immersive experience to the users'. This technology provides novelty by combining real and virtual world and registering it to $3 \mathrm{D}$ reality [11]. The advancement in AR has introduced new teaching methods which enhances the interest and class participation of students [12]-[17]. Recent studies show that students engaging in such interactive ways of learning have great positive impact on their education [14].

Recent studies proposed by Latvian teachers tells us that old methods of teachings have reduced the interest and concentration level of the students [15]. The overall results in Fig. 1 shows that the majority of students have decreased interest in the current methods of teachings [15].

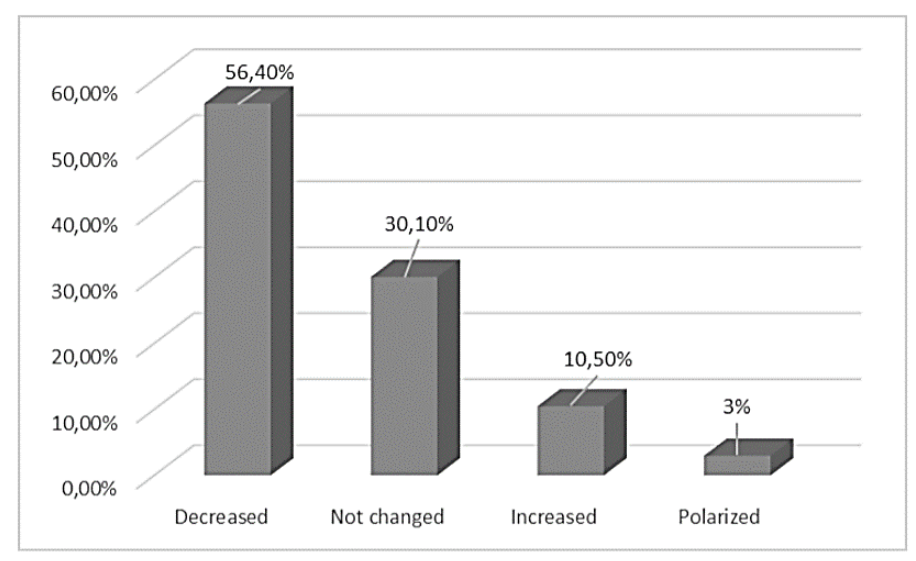

Fig. 1. Opinion of Teachers about Changes of Students' Learning Motivation [15].

In addition, some studies have been conducted to assess the usability of augmented reality in educational field. In one of the study, D.L Hakim et al. [10] concluded that AR has a significant impact as a learning media and has greatly affected students' motivation and learning outcomes. Annafi A et al. in [10] reviewed and collected data from 30 articles published in the past 10 years. The articles reviewed the cognitive, affective, and psychomotor aspects that include students' cognitive skills, learning abilities, understandings, motivation, responses, attentiveness, involvement, and outcomes towards any study material [10].

In another review, Nicholas Pellas et al. [16] analyzed the literature on AR with Game-based Leaning (ARGBL) approach. The ARGBL system was developed while keeping in view the advantages, disadvantages, instructional affordance and effectiveness across various primary and secondary education [16]. A detailed methodology proposed by Kitchenham's paper published in 2007 was adapted for the purpose of systematic review [16]. The journals were selected through inclusive and exclusive criteria and then the data was catego- 
rized and analyzed. It was concluded that the ARGBL usage has significantly increased for teaching Science, Technology, Engineering and Mathematics (STEM) in the past few years.

In another review, Marina Ismail et al. [18] provided a comprehensive review of existing studies on the use of Kinect device in education and rehabilitation. A total of 16 studies were collected, analyzed and organized in a detailed order [18]. It concluded that Kinect-based systems are beneficial in providing e-learning environment and interactive experiments [18]. The outcome of these reviews demonstrates an overall positive impression and serves as a motivational factor for further analysis on AR as a learning tool. Similarly, in this paper a systematic review of 10 research papers is carried out focusing on AR-based educational systems.

Our Contributions are as follows: i) A detailed review of the latest AR-based learning systems. ii) Developed an algorithms to simulate the particle physics experiments i.e. proton-proton collision and Higgs field. iii) Designed an ARbased learning system to demonstrate the particle physics experiments with an immersive experience.

\section{LiterATURE REVIEW}

In this section, a detailed review of recent research papers is conducted to show the impact of AR on modernizing the classroom learning. The goal of this review is to lay out a comprehensive analysis about the impact and findings of recently proposed systems in the field of education. The steps taken to select the qualitative research papers are as follows:

1) The data is collected from academic journals of MDPI, IEEE ACCESS, Springer, International journal of Engineering and Technology, Elsevier, Canadian center of Science and Education, International journal of Geographical Information Science, Journal of Physics, and Hindawi which are published in the years from 2015 through 2020.

2) Numerous keywords such as "Kinect sensor and AR", "AR in education", "Impact of AR in educational field", "Lab based experiments on Kinect sensor", "Interactive wall and floor in classrooms", "Advantages of using AR in classrooms", "AR effects on children learning" were used to find the most relevant articles.

3) Approximately 40 articles were selected and analyzed keeping in view the title, abstract and keywords of the paper. Total of 11 studies were identified as the most relevant to the topic and hence were extensively reviewed.

The summary-based analysis of each study is given below:

- Franca Gorzotto et al. [19] created a Magic Room using projector, Kinect sensor, several smart objects and Unity 3D software for children with Neural Developmental Disorder (NDD). The games developed, in this paper, detect the children's behavior as they interact with the multimedia content in the Magic Room [19]. The experiments proved to elicit functional performances, social behaviors, and emotional responses. The authors concluded that further empirical research is needed in this area as the experiment was limited to health care [19], [20].

- Plamen D. Petrov et al. [12] analyzed the effect of AR on students' learning performance in STEM education. This experiment uses ZSpace which is an all-in-one AR system comprising of virtual reality monitor and a computer. It combines AR and VR to create an immersive and interactive experience as shown in Fig. 2 [12]. The experiment was carried out on 80 participants and a significant difference was observed in students' understanding as compared to the traditional system [12]. In conclusion ZSpace introduces high level of personalization and helps improve the understanding of students. It allowed students to explore and practice without worrying about financial (supporting lab equipment) or ethical (animal injury in biology lab) issues [12].
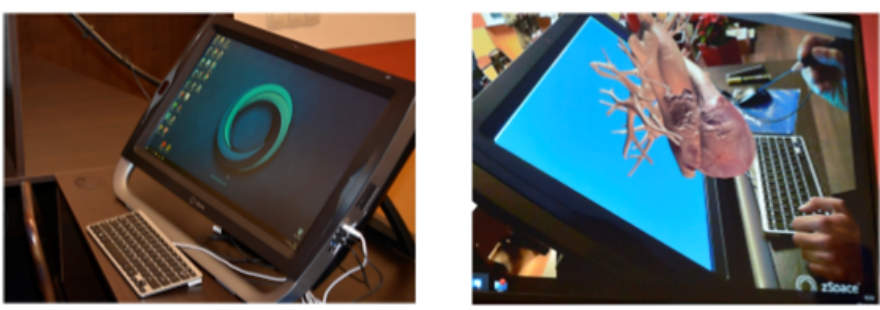

Fig. 2. ZSpace as an AR Tool for STEM Education [12].

- Lidice Haz et al. [3] implemented a Kinect-based multimedia system for children of primary schools for increasing classroom participation. This system was developed in a cascaded model consisting of levelbased design with incrementing difficulty. The models enabled students to add, subtract, complete words and complete sentences. A survey indicated that $87 \%$ of students preferred this method of learning [3].

- Nak-Jun Sung et al. [21] investigated the applications of the Physics using AR. A video see-through method is used to construct an AR environment by using Kinect V2 sensor. The experiment uses soft body simulator version MSS (Mass Spring System) because of its high simulation accuracy and speed. The AR experiment first combines a real time video stream with a soft body simulation as shown in Fig. 3. Then, it creates several objects with various material properties by changing the object motion through simulation as shown in Fig. 4. In conclusion, a survey showed that $93 \%$ of responders were in favor of teaching the Physics using this simulator [21].

- Tamas Matuszka et al. [22] developed a gesturecontrolled educational gaming system. Authors reduced the cost of the system by using deep learning method. The algorithm first detects the object and uses "sliding window method" gesture collection. Keras and Tensorflow were used as deep learning backend. In conclusion, ordinary camera with the proposed algorithm provided similar results as depth-camera based system. 


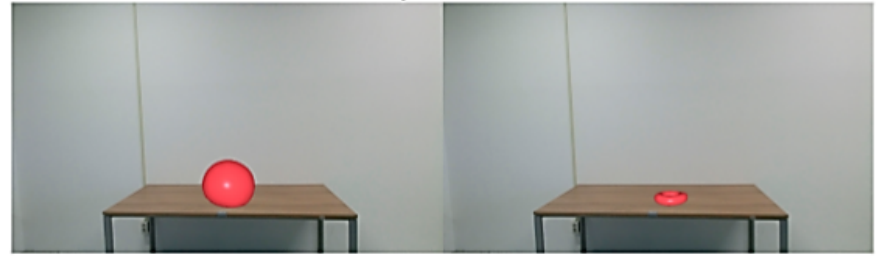

Fig. 3. Combined System of Soft Body Simulator and Video Stream obtained from Kinect Device [21].

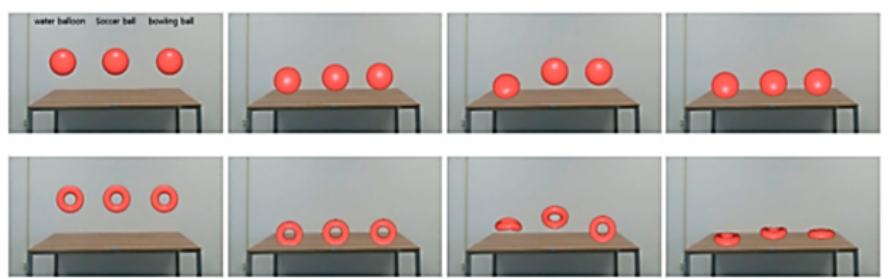

Fig. 4. Result of Simulation of 1st Scene Comprising of Sphere Model [21].

- $\quad$ Raul Lozada-Yanez et al. [23] designed a Kinect-based AR Math Learning System (KARMLS) for increasing student performance in mathematics. It involved 29 third-grade children from Riombamba city, Ecuador. The system comprises of sumar (addition), ordeanr (arranging shapes in order) and parear (making pairs). The Fig. 5 shows student interaction with KARMLS using wave gesture. It was concluded that the system had a positive impact on students and was more effective on low grades securing students [23].

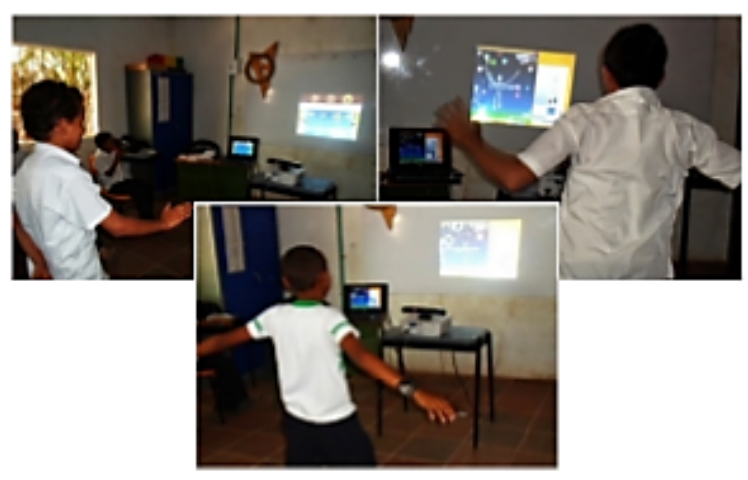

Fig. 5. Natural Interaction between End User and Kinect based Prototype [23].

- Corey Pittman et al. [24] explored the utility of AR for knowledge of the Physics in the classroom. A PhyAR prototype was developed using Unity3D and mixed reality toolkits with Microsoft HoloLens [24]. Coulomb's Law, elastic collision, parallel circuits, volume etc. were demonstrated using virtual objects in the physical space as shown in Fig. 6. Fifteen participants were gathered to explore each concept using prototype and then fill a questionnaire for feedback [24]. The students' response was positive but
HoloLens being a head worn device restricted the physical world interaction. Feedback from the students emphasized on adding interaction with real world objects [24].

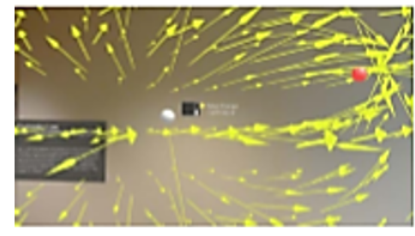

a. Coulombs Law

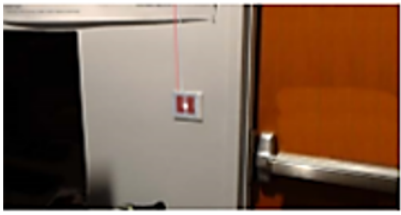

c. Parallel circuit

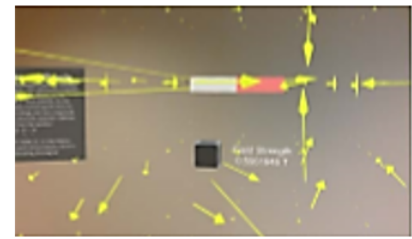

e. Magnetic Field

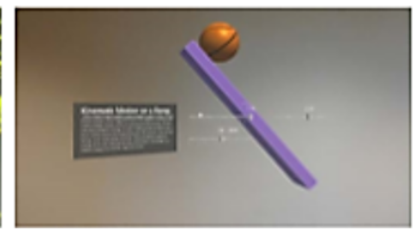

b. Elastic Collision

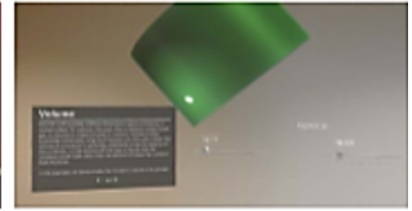

d. Volume

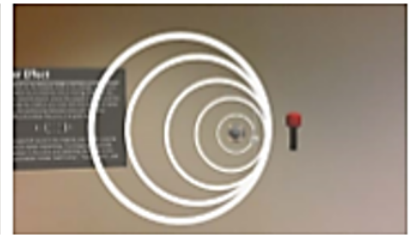

f. Doppler Effect
Fig. 6. Presentation of Some Physics Topics shown on PhyAR Application on Microsoft Hololens [24].

- Mingshao Zhan et al. [25] studied the recent developments in game based virtual educational laboratories using Kinect sensor. Kinect device is used to scan the real-world data and a map is created to implement a virtual laboratory. The RANdom SAmple Consensus (RANSAC) algorithm is used for shape detection as shown in Fig. 7 [25]. In this way, integration of real materials with natural materials have been observed and presented [25], [26]. Microsoft Kinect sensor proved to be realistic and affordable for virtual environment modeling, human-computer interface, and hardware interface implementation.

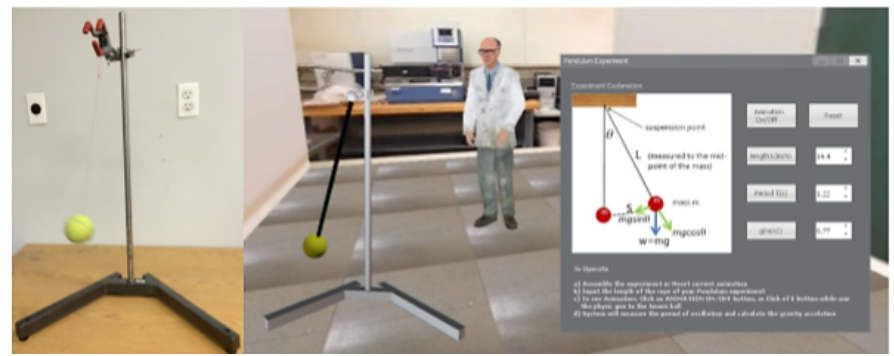

Fig. 7. Experimental Setup of Foucault Pendulum (Left); Foucault Pendulum Experiment Implementation in Game-based VE (Right) [25].

- Mingliang $\mathrm{Xu}$ et al. [27] provided a Kinect-based system for physical education of trainees (children) without trainers. For training this system, Hierarchical 
Hidden Markov (HMM) based algorithm was used. It allowed trainers to develop customized training paths for each individual trainee. This method significantly enhanced the effects of physical training in the absence of trainers [27].

- Yi-Hsing Chang et al. developed a Kinect-based English learning system [28]. They integrated Kinect as the interaction technique with theories of situated learning and attention, relevance, confidence, and satisfaction (ARCS) model [28]. This system enables to plan and design the learning activities as per situated learning [28]. The system provides virtual environment which helps to achieve spatial and physical experience, assisting learner's engagement, and enhancing learning motivation as shown in Fig. 8. The authors concluded proposed system improved students learning motivation [28].

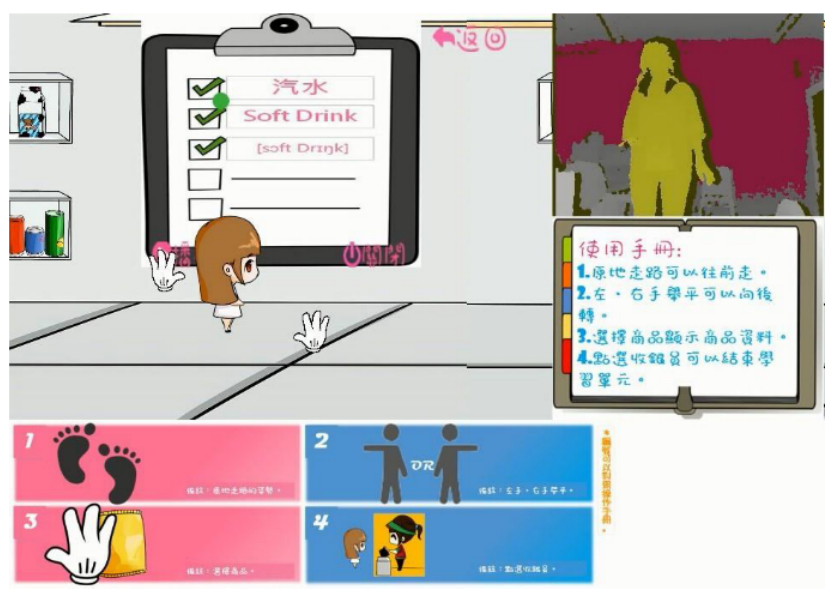

Fig. 8. English Learning system using ARCS Model) [28].

- Maria Cristina Costa et al. presented a mobile augmented reality based application called PlanetarySystemGo which is a location-based game to promote learning about the universe [29]. The architecture of the system is divided into three components: platform server (manages all data in the system), Web application (the assessment of learning outcomes with a back office) and mobile app (created using unity $3 \mathrm{D}$ to provide dynamic environment to create $\mathrm{AR}$ content) as shown in Fig. 9. Several surveys including questionnaires were conducted with primary school students and teachers. According to the results, the application enhances the students' interest to learn about solar system and keep them engaged [29].

The above studies are further analyzed and categorized into major contribution and limitation provided by authors. It provides all-inclusive information about how AR with the help of Kinect sensor has changed the way of learning. Data is taken exclusively from the said articles and has been compared in Table I.

\section{PROposed System OVERVIEW}

This section presents the development of proposed ARbased learning system. The system is developed to demonstrate
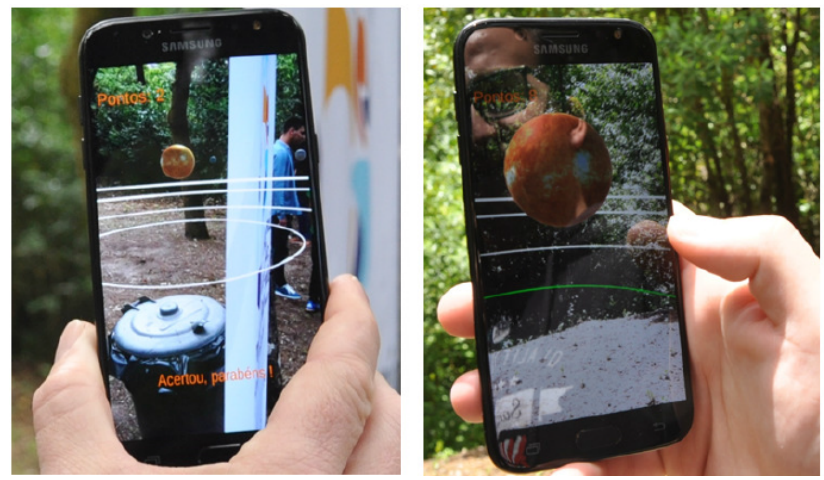

Fig. 9. Mobile App Showing Hunting Orbits and Planets.) [29].

the experiments of particle physics. The proposed system is developed to simulate the proton-proton collision and Higgs field. The algorithms are developed using development software Unity 3D, Microsoft Kinect V2, a projector, C\# (Csharp) language and Visual Studio IDE. Kinect sensor was interfaced with Unity 3D to enable interaction of objects with the environment. The Microsoft SDK for Kinect sensor is used to track the body with the help of its infrared-based depth camera. It accurately captures the real time 3D scene and generates the built-in skeletal using customized software [30], [31].

In the proposed system the actions performed by human body are acquired and processed by the Kinect Sensor. Then these actions are evaluated on basis of the developed algorithms and resultant output is delivered via Projector. The proposed system is shown in Fig. 10.

The developed environment is projected on the floor providing the real-time interactive learning experience. The algorithm developed for Higgs field allows the user to visualize their reflection inside an particles filled environment.

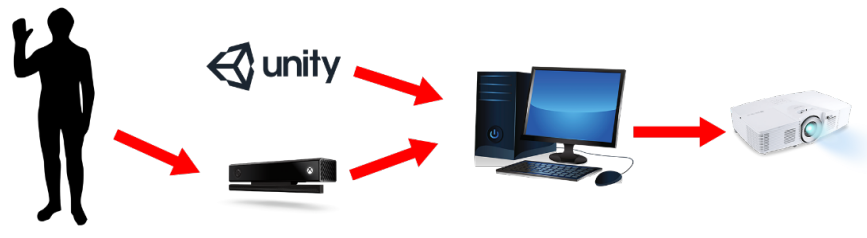

Fig. 10. The Proposed System.

Algorithms are developed to demonstrate the particle behavior such as proton-proton collision effect and Higgs field.

Proton collision algorithm shows the generation of new particles as a result of proton-proton collision. In this algorithm, the environment is designed where students can play a football game in which the footballs are supposed as protons.

\section{PROPOSED SySTEM IMPLEMENTATION}

\section{A. Proposed Algorithm for Proton-Proton collision}

The collision of protons is possible through LHC (Large Hadron Collider) tunnel, which is particularly a particle accelerator owned by CERN laboratories [32]. This tunnel allows 
TABle i. Details about Studies Related to AR Technology in Educational Field.

\begin{tabular}{|c|c|c|}
\hline Source & Major Contribution & Limitations provided by authors \\
\hline [19] & $\begin{array}{l}\text { Magic room developed in this paper has a strong potential as a learning } \\
\text { environment for children with Neurodevelopmental Disorder (NDD) }\end{array}$ & $\begin{array}{l}\text { Regular update of the content in areas like communication, Psychomotor, } \\
\text { emotion, and cognition is required }\end{array}$ \\
\hline [12] & $\begin{array}{l}\text { The data obtained from performing experiments revealed that the integration of } \\
\text { AR allows students to explore, practice and interacts with Science, Technology } \\
\text { Engineering and Mathematics (STEM) content with an effective way. }\end{array}$ & Price of the overall system is 7000 dollar which makes it costly \\
\hline [3] & $\begin{array}{l}\text { It concludes that Kinect sensor with multimedia technology facilitates the } \\
\text { teaching and learning process through an attractive and motivating environment }\end{array}$ & $\begin{array}{l}\text { Physical interaction should be enabled for more enhanced and learning experi- } \\
\text { ence. }\end{array}$ \\
\hline [21] & $\begin{array}{l}\text { According to the findings, the proposed simulator helps to teach the Physics } \\
\text { effectively due to more realistic representation of complex processes }\end{array}$ & $\begin{array}{l}\text { Requires more realistic representation of certain content and high specification. } \\
\text { Additional equipment is required to expand the project. }\end{array}$ \\
\hline [22] & $\begin{array}{l}\text { It has been found that a cost-effective monocular camera-based gesture recog- } \\
\text { nition method can ensure similar level of recognition accuracy as depth-camera } \\
\text { based solutions }\end{array}$ & Limited gesture recognition as compared to Microsoft Kinect. \\
\hline [23] & $\begin{array}{l}\text { The outcome of experiments suggested that there is a significant improvement in } \\
\text { grades of students using Kinect Based AR Math Learning System (KARLMS) }\end{array}$ & Better visual graphics and interactivity should be enabled for higher accuracy. \\
\hline$[24]$ & $\begin{array}{l}\text { The participants' evaluation revealed that the users desired to see such } 3 \mathrm{D} \text { AR } \\
\text { content in the Physics. }\end{array}$ & $\begin{array}{l}\text { Limited field of view, integration of physical objects, and support of environment } \\
\text { around the user are required. }\end{array}$ \\
\hline [25] & $\begin{array}{l}\text { According to findings of the research, Microsoft Kinect based educational } \\
\text { VR laboratory proved to be efficient, realistic, and affordable as compared to } \\
\text { traditional approaches }\end{array}$ & Integration of more tracking algorithms into DAQ software package is required. \\
\hline [27] & $\begin{array}{l}\text { User study evaluation contributes to the research that the effect of Kinect-based } \\
\text { training method is much better than the traditional video-based method. }\end{array}$ & Better visual graphics and interactivity should be enabled for higher accuracy. \\
\hline [28] & $\begin{array}{l}\text { This system helped students to learn English effectively through integrating } \\
\text { Kinect with situated learning and ARCS model. }\end{array}$ & $\begin{array}{l}\text { The system seizes to operate during experiment. Furthermore, instructions were } \\
\text { too descriptive having small font size, making them unreadable. }\end{array}$ \\
\hline [29] & $\begin{array}{l}\text { it has been noted that the PlanetarySystemGo platform has great potential in } \\
\text { serving as an informal and formal learning environment about the solar system } \\
\text { for all students. }\end{array}$ & $\begin{array}{l}\text { Some technological hindrances were observed such as instability due to GPS } \\
\text { coordinates and inaccurate gyroscope reading. Also, the information content } \\
\text { should be more enhanced and upgraded such as more planetary system need to } \\
\text { be introduced. }\end{array}$ \\
\hline
\end{tabular}

the particles to travel approximately at the speed of light (3 meters per second less than the speed of light), and gain higher energies. The number of particles dispersed after the protonproton collision is directly dependent upon the speed and energy of the proton. The scientists observe the new particles by colliding protons at a higher velocity. The new particles are smaller as compared to protons and have different properties altogether [32].

The proposed system simulates the LHC tunnel to visualize the generation of new particles after collision of protons. This system is developed in the form of a game to enable the users' interaction in the particle acceleration, collision and generation in a fun and interesting way. Using this system students can observe that the amount of new particles generated is directly proportional to the velocity of protons.

The proposed particle behavior system follows the String Theory / String Model. It states that, upon collision, two protons divide into particles resembling a string [33]. The distribution of the particles according to String model follows the Equation (1):

$$
\mathbf{x}_{1}=(1-y) \mathbf{x}_{o}
$$

where, $\mathbf{x}_{1}$ represents the number of particles created after collision, $\mathbf{x}_{o}$ is the energy applied by the kick, whereas $y$ is the fraction of energy carried by string (distributed particles). The value of $y$ exists between 0 and 1 depending upon the distribution function. Equation (1) can be further extended for the number of new particles.

$$
\mathbf{x}_{n}=\left(1-y_{a v}\right)^{i} \mathbf{x}_{o}
$$

Where $\mathbf{x}_{n}$ is the residual energy of the string after $i^{\text {th }}$ rank of distribution, $y_{a v}$ is the average value of $y$, and $i$ is the average multiplicity of the produced particles which can be calculated using Equation (3) and Equation (4).

$$
i=\ln \frac{\mathbf{x}_{o}}{\mathbf{x}_{n}} \beta
$$

where,

$$
\beta=\frac{-1}{\ln \left(1-y_{a v}\right)}
$$

The proposed simulated environment allows two users to kick the protons (simulated as footballs) in a real-time. The momentum of the kick determines the speed of protons. Users can visualize the generation of new particles when protons collide.

Taking our proposed system into consideration, the external force applied (kick) is responsible for increasing the length of the string/number of distributed particles. The higher the force, the more the production of particles. 


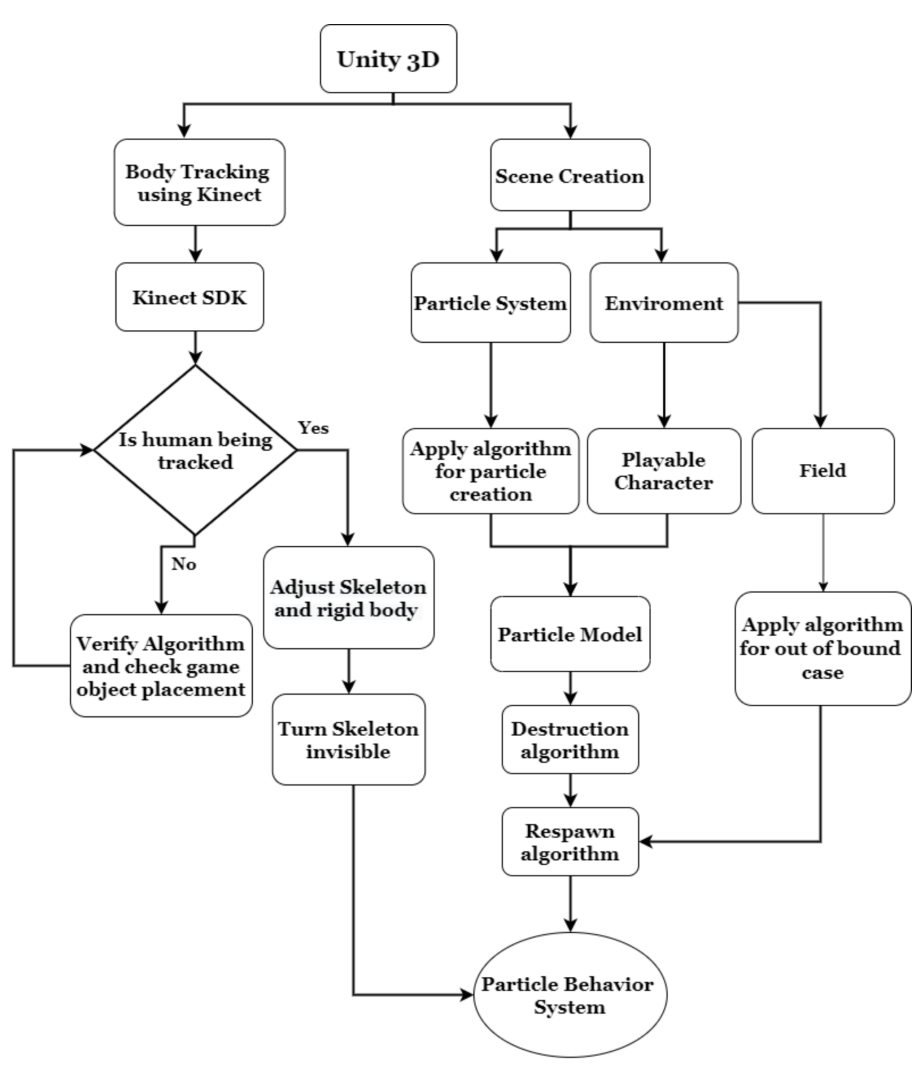

Fig. 11. Particle Behavior System Flow chart.

1) Implementation of Proton-Proton Collision: Firstly, we created an empty 3D project inside Unity 3D, allowing us to develop a system in 3D environment. After that Microsoft Kinect V2 was connected to PC via adapter and its SDK was imported inside Unity. Methods available in SDK were used to create scripts that allowed human joints tracking. The joints of tracked bodies were created using 3D objects like spheres, as displayed in Fig. 12. These objects were also provided with rigid body and collider modules through scripting. Rigid body module allows the object to have properties like gravity, force, acceleration etc. Whereas collider module allows the object to collide with any other surface. If the collider module is not enabled, the objects cannot collide and pass through one another.

A scene was created in which the system was to be developed. Firstly, the environment was developed using 3D objects. The playable characters (Protons) were created using two Spheres and the Field (platform) was designed using multiple cubes arranged accordingly as displayed in Fig. 13. Playable characters were provided with rigid body and collider modules, whereas only collider module was provided to the field. A script was attached to the field objects so that the spheres return to their original position whenever they were out of bounds.

The built-in particle generation system of Unity 3D was used for particle generation as shown in Fig. 14. This system helps generate particles of various sizes and quantities. It also helps to create trails behind each particle. A C\# script is used to simulate the number of particles generated using the speed

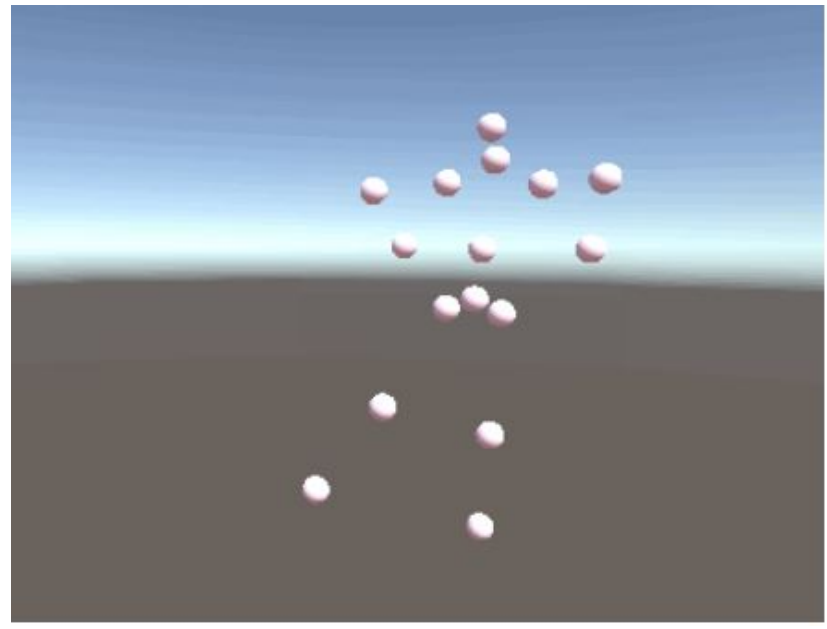

Fig. 12. Skeleton Tracking using Kinect.

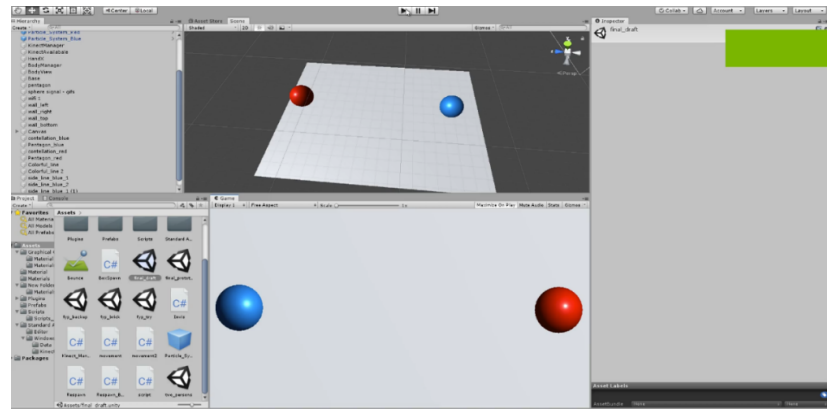

Fig. 13. Two Spheres Representing Two Protons.

of spheres, using the Equation (2) of String Model.

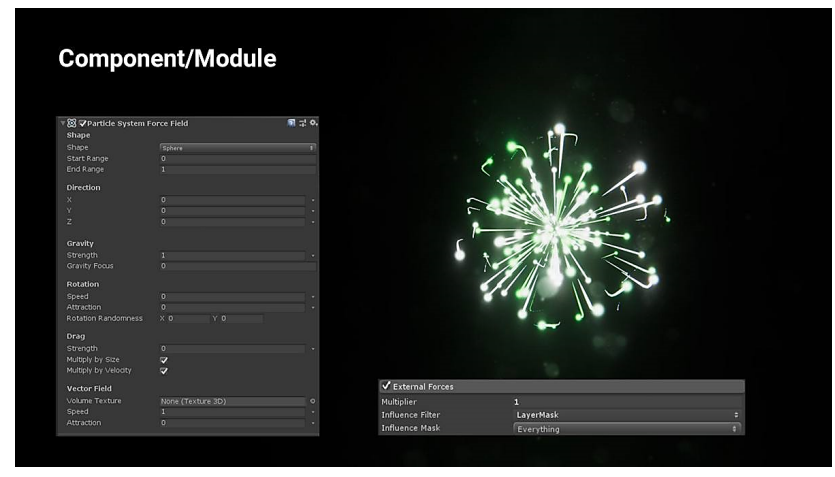

Fig. 14. Unity’s Particle Generation System.

Graphics were embedded on each of the game object and were created using Adobe Photoshop and Adobe Premiere Pro as displayed in Fig. 15. The system was named "Particle Model" and was provided with scripts that allowed the destruction of spheres upon collision. This script allowed the spheres to be destroyed upon collision and generates particles as shown in Fig. 16. Another script was attached to the model that allowed the particles to respawn at original positions. Speed bar is included in the environment to observe variation in speed of the spheres. Particle model is finalized by overlapping tracked bodies over the particle model while converting the skeleton 


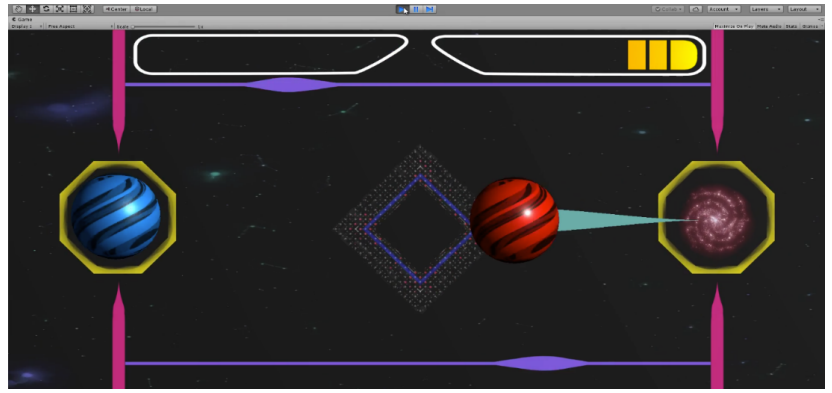

Fig. 15. Graphical Interface of Proton-Proton Collision System on Unity 3D.

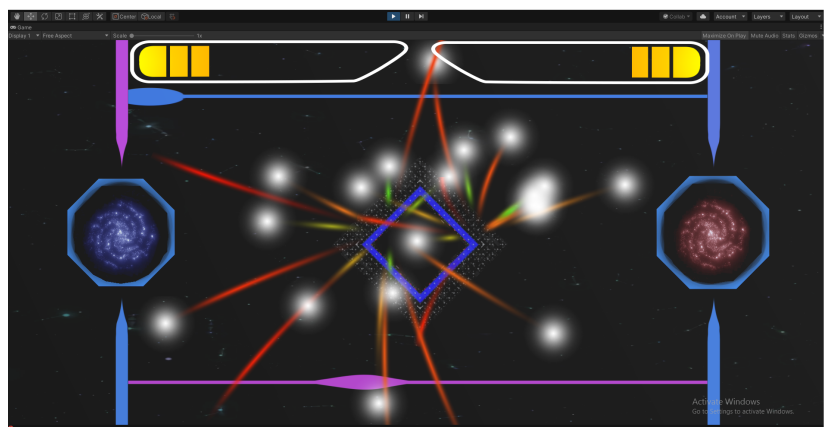

Fig. 16. Graphical Representation of Particle Generation after Collision.

to be invisible for better visual experience as displayed in Fig. 17. A flow chart of proposed algorithm is shown in Fig. 11.

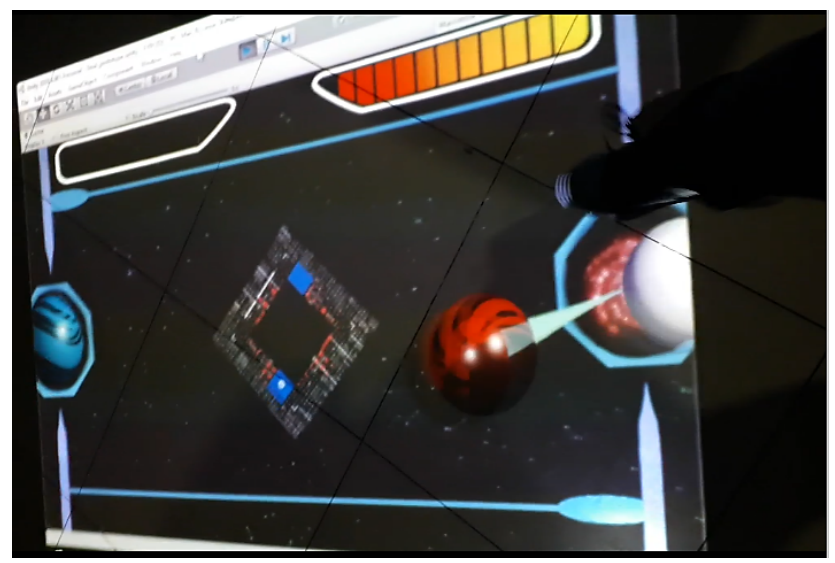

Fig. 17. Practical Testing of Proton-Proton Collision System.

\section{B. Higgs Field}

Higgs Field is a field of energy that exists everywhere in the entire universe [34], [35]. The particle known as Higgs boson attracts other particles towards itself to gain mass [36][38]. Work on the Higgs field and Higgs boson started in 1964 [39], [40]. The theory of particle was first introduced by PW Higgs in 1964, describing the existence of a particle having a mass of $125 \mathrm{GeV}$ (giga-electron volt) [40]. On 4th of July, 2012, by collaboration of Compact Muon Solenoid and Atlas, the Higgs Boson was first discovered using the LHC tunnel [34], [37].
The proposed system enables to visualize the human body in the presence and absence of the Higgs Field. Thus, explaining the concept of Higgs field and Higgs Boson. The simulated environment shows that when user stands inside the Higgs field, Higgs Boson particles surrounds the user. On a contrast, when body is outside the Higgs field, the particles do not interact with the user. This allows users to visualize that how Higgs Boson interacts with other atoms.

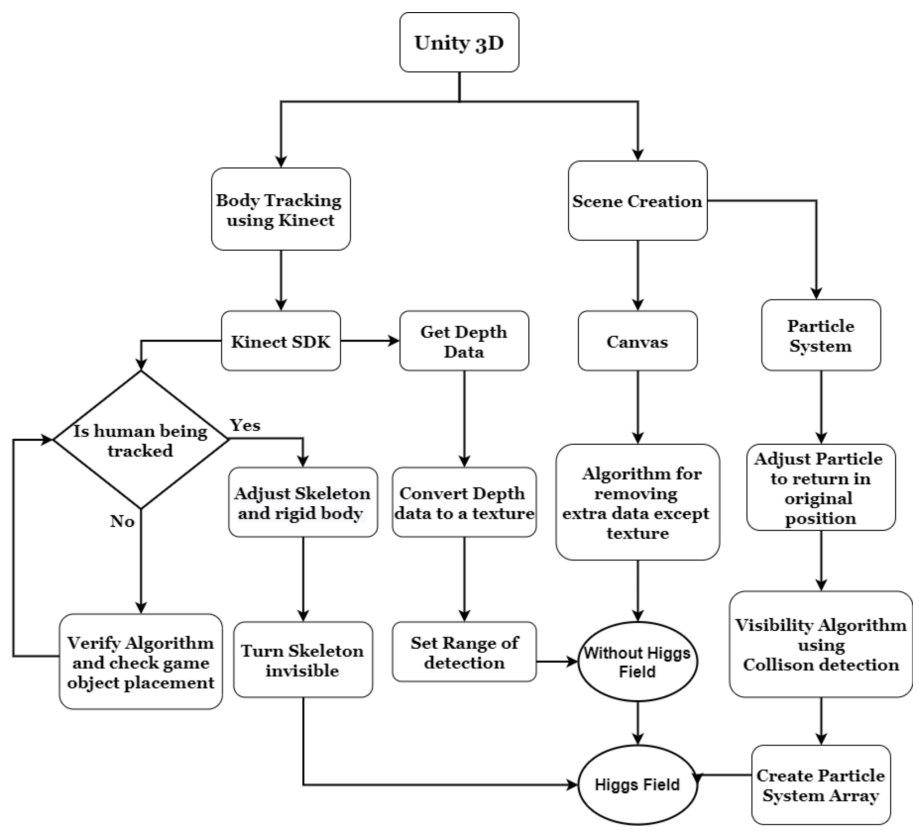

Fig. 18. Higgs Field Flow Chart

1) Implementation of Higgs Field: Firstly, we created an empty 3D project inside Unit 3D, allowing us to develop a system in 3D environment. The same procedure was used to track and create human joints, as in the particle behavior system and it was also made invisible. The script was created to use the depth camera. Data from the depth camera is stored in ushort datatype which cannot be visualized. So, for visualization, we convert depth data from ushort to Texture2D format. A limiter is set which allowed only for the detection of object inside specified z-axis.

A scene was created in which the system was developed. An algorithm was applied to the canvas which allowed it to only make Texture2D datatype visible. The Texture2D enabled us to visualize the behavior of Higgs Boson with other particles in the absence of Higgs field as shown in Fig. 19.

Particle System is created and adjusted to allow the particles to start from outside and return to their respective origins. A script was created and applied to particle system that helps users' to visualize the effect of particle system whenever any object collides with it. An array of particle systems was created using the adjusted particle system and was aligned with the canvas as shown in Fig. 20. The implementation of particle system allowed us to visualize the behavior of Higgs Boson with other particles in the presence of Higgs field. Fig. 18 shows the flow chart of proposed algorithm. 


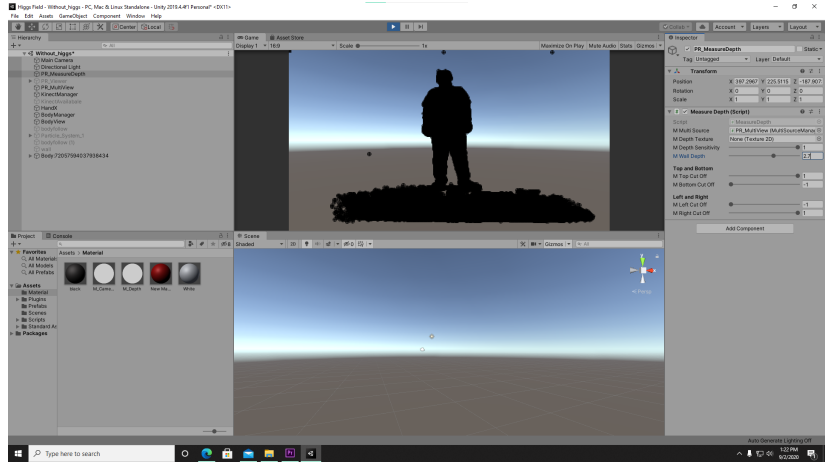

Fig. 19. Depth Camera Data (ushort datatype) converted to Texture2D and implemented on Canvas.

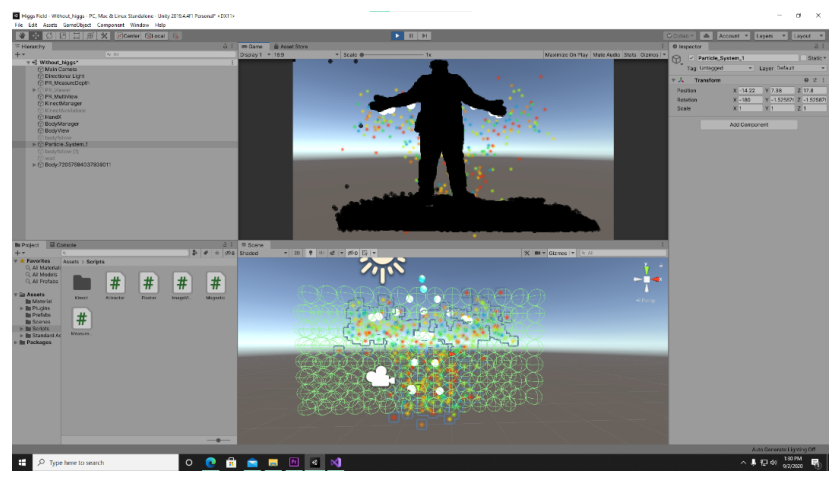

Fig. 20. Visualizing Higgs Field (High Resolution of Canvas).

\section{REsults AND Discussion}

This section presents the qualitative and quantitative evaluation of the proposed system. Also the qualitative analysis of the latest AR based learning systems is presented.

\section{A. Qualitative Analysis}

The qualitative analysis is based on cost, product originality, scalability, flexibility and market demand as shown in Table III. These are few factors for a product to gain a place in the market and customer attention. The cost factor shows the cost per module of the system. Product originality shows that whether the system developed by the authors is a new product or a revamp (improved version of a previous product). Scalability shows us whether the system can be integrated and scaled towards other fields. Flexibility tells us whether the product or system created can be upgraded later in the future or not. Market Demand is a biased factor which solely depends upon the region of sale. We have considered the sale or outreach of these products on the basis of market trends in Pakistan. The qualitative results suggest that the proposed learning system is highly comparable with recently developed AR based learning systems.

1) Estimated Cost: The proposed system cost around 631 USD. The cost of Projector, Kinect Sensor, and the developed software are the main components. Table II shows the cost of the proposed system.
TABLE II. Cost of Proposed System.

\begin{tabular}{|c|c|c|}
\hline S.No & Items/materials required & Cost in USD \\
\hline 1 & Kinect sensor & 156 \\
\hline 2 & Projector & 437 \\
\hline 3 & Software development and installation & 38 \\
\hline \multicolumn{2}{|c|}{ Total cost } & 631 \\
\hline
\end{tabular}

\section{B. Quantitative Analysis}

The quantitative analysis is based on a survey as suggested in [21], [29]. A questionnaire was developed for analyzing the effectiveness of the proposed system. Questionnaire shown in Table IV is similar to the questionnaires developed in [21], [29]. In [21], [29], a post-study questionnaire involving scale-based and free-response questions was taken from fifteen participants aged between 21-31 years. Whereas in our study, a total of 20 college students aged between 19 and 22 years participated.

The survey was conducted following the similar pattern as discussed in literature [2], [21], [24], [27], [29]. Firstly, the participants were briefed about the proton-proton collision effect and Higgs field through conventional method of teaching. Then, the proposed AR-based system was used to demonstrate the same concepts. The proposed system allowed the students to interact with the environment and visualize its results. The understanding was made easy, fun-to-learn and interactable.

The survey was performed in a group of 5 students each lasting 20 minutes.

The results attached in Fig. 21 were analyzed on the basis of the methods used in [21], [29]. It mainly focuses on the effectiveness of the AR based learning systems in learning process.Whereas, Fig. 22 shows the score of each questions. Similarly, the result of first four questions focuses on the improvements in the understanding of topics. Whereas, the Q5 result shows the response of students about recommendation of proposed system as shown in Fig. 23. It was concluded that $85 \%$ of the students recommended the proposed system for effective learning.

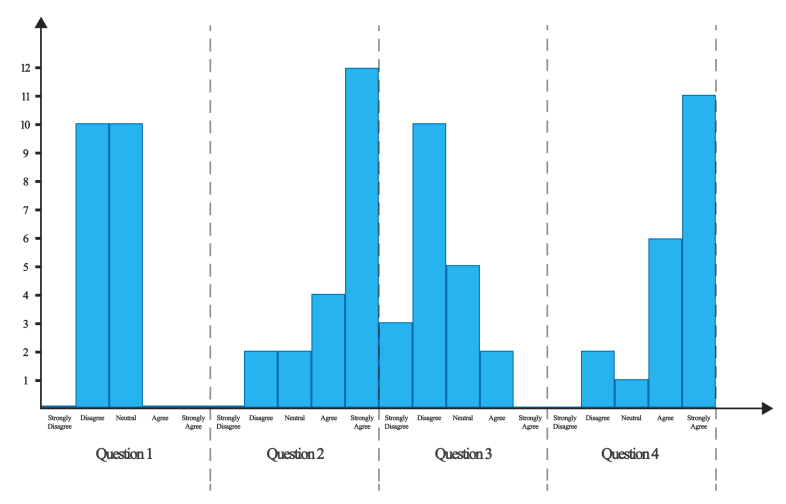

Fig. 21. Survey Results.

\section{Limitations of the Proposed System}

Following are the limitations of the proposed system: 1. The total horizontal and vertical field view of Kinect sensor 
TABle III. Qualitative Analysis of Recent AR based Learning Systems and Proposed System.

\begin{tabular}{|c|c|c|c|c|c|}
\hline Source & Cost in USD & Product Originality & Scalability & $\begin{array}{c}\text { Flexibility (future } \\
\text { work) }\end{array}$ & $\begin{array}{c}\text { Market Demand } \\
\text { (Value) }\end{array}$ \\
\hline$[19]$ & $5 \mathrm{k}$ & New product & High & High & Medium-high \\
\hline$[12]$ & 950 & Revamp & High & High & Medium-high \\
\hline$[3]$ & 350 & Revamp & Medium & High & Low-Medium \\
\hline$[21]$ & 650 & Revamp & Medium & Medium & Low-to-medium \\
\hline$[22]$ & $1.3 \mathrm{~K}$ & New product & High & High & Medium-High \\
\hline$[23]$ & 625 & Revamp & Medium & Medium & Low-to-medium \\
\hline$[24]$ & 625 & Revamp & Low & Medium-Low & Low \\
\hline$[25]$ & 625 & Revamp & Medium & Medium-High & Medium-high \\
\hline$[27]$ & 375 & Revamp & Medium & High & Medium-High \\
\hline$[28]$ & 375 & Revamp & Medium & High & Low-Medium \\
\hline$[29]$ & 63 & New product & Medium & Medium-High & Medium-High \\
\hline $\begin{array}{c}\text { Proposed } \\
\text { System }\end{array}$ & 631 & New product & High & High & Medium - High \\
\hline
\end{tabular}

TABLE IV. Proposed System Survey (5 Multiple-Choice Questions).

\begin{tabular}{|c|c|}
\hline Number & Question Details \\
\hline Q1 & It is easy to understand proton-proton collision effect without AR-based demonstration \\
\hline Q2 & I found AR-based demonstration very helpful in understanding the proton-proton collision experiment \\
\hline Q3 & It is easy to understand the concept of higgs field without AR-based demonstration \\
\hline Q4 & I found AR-based demonstration very helpful in understanding higgs field experiment \\
\hline Q5 & I highly recommend this product in education which can help student to understand complex problem \\
\hline
\end{tabular}

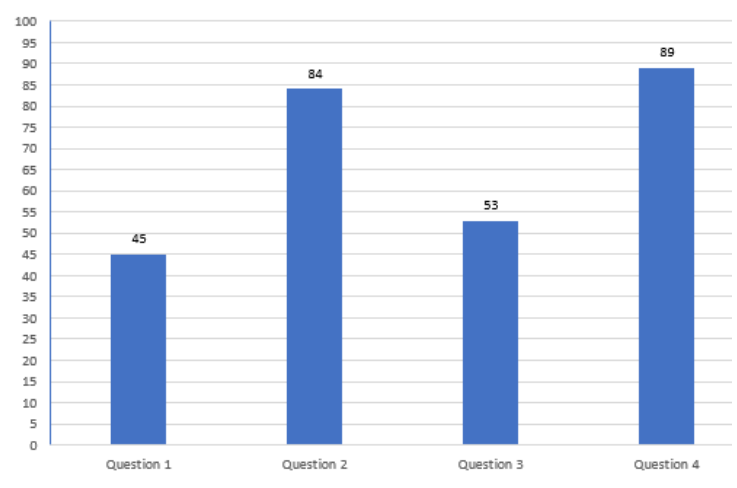

Fig. 22. Results of Individual Questions.

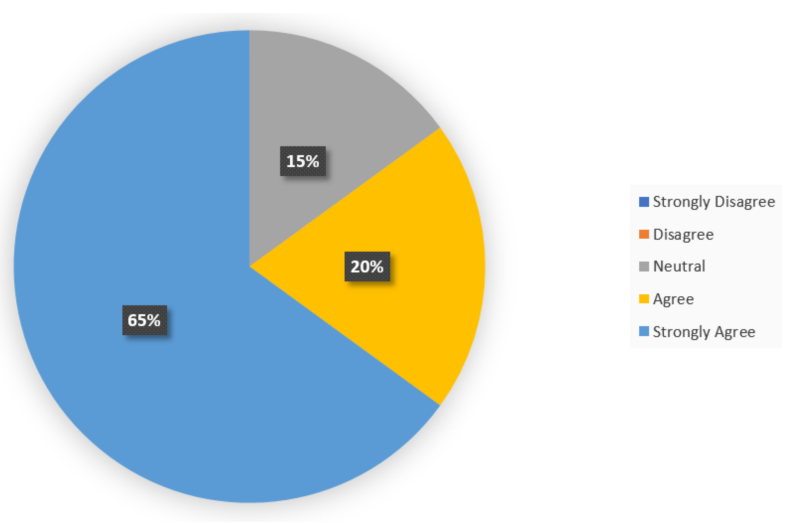

Fig. 23. Results of Project Recommendation Question (Q5). is 70 and 60 degrees respectively. Hence the proposed system is subjected to a limited space. In order to cover entire room (360 degrees), more Kinect sensors are needed to be linked together.

2. The proposed system can smoothly detect up to two people at a time. Hence putting a constraint on certain application where more than two people needs to be detected at the same time. Using more Kinect sensors can also solve this issue.

3 . If the user performs a fast movement, there is an issue of delay. Hence depth camera requires some extra time to detect each movement correctly. This issue can be resolved using Graphical Processing Unit with high memory specifications.

4. The Kinect sensor has to be fixed and aligned with the position of users for smooth experience of users.

5. The Kinect sensor has a specific detection range of around 10 meters.

6. The proposed system works better in a room where there is minimal or no sunlight.

\section{CONCLUSION}

In this research paper, the detailed review on latest advancement in AR-based learning systems is presented. The detailed review is based on major contributions and limitations provided by authors. It is concluded through intensive review that AR-based learning systems are effective to incorporate $21^{\text {st }}$ century skills such as critical thinking, creativity, communication, collaboration, innovation and problem solving. Furthermore, an AR-based learning system is developed to demonstrate the particle physics experiments. The proposed system simulates the proton-proton collision and Higgs field 
using unity 3D software. The Proton-Proton collision algorithm simulates the generation of new particles when protons collide with each other. It demonstrates that number of particles generated depends on energy of the colliding protons. Whereas, Higgs field simulation highlights the effect of Higgs Boson inside and outside Higgs field. The proposed system is developed using unity $3 \mathrm{D}$ software and then interfaced to Kinect sensor for immersive experience. Then, the qualitative analysis of the proposed system and latest AR based learning systems is presented. Finally, the quantitative analysis of the proposed system is conducted. Overall, the results suggest that $85 \%$ of the participants recommended the proposed learning system.

\section{REFERENCES}

[1] J. Egger and T. Masood, "Augmented reality in support of intelligent manufacturing - A systematic literature review," Comput. Ind. Eng., vol. 140, p. 106195, 2020, doi: 10.1016/j.cie.2019.106195.

[2] P. Fraga-Lamas, T. M. Fernández-Caramés, Ó. Blanco-Novoa, and M. A. Vilar-Montesinos, "A Review on Industrial Augmented Reality Systems for the Industry 4.0 Shipyard," IEEE Access, vol. 6, pp. 13358-13375, 2018, doi: 10.1109/ACCESS.2018.2808326.

[3] L. Haz, Y. Molineros, E. Vargas, and A. Davila, "Multimedia system Kinect-based. Learning experience for children of primary school," CEUR Workshop Proc., vol. 2486, pp. 295-308, 2019.

[4] S. B. Adikari, N. C. Ganegoda, R. G. N. Meegama, and I. L. Wanniarachchi, "Applicability of a Single Depth Sensor in Real-Time 3D Clothes Simulation: Augmented Reality Virtual Dressing Room Using Kinect Sensor," Adv. Human-Computer Interact., vol. 2020, 2020, doi: 10.1155/2020/1314598.

[5] G. Dini and M. D. Mura, "Application of Augmented Reality Techniques in Through-life Engineering Services," Procedia CIRP, vol. 38, pp. 14-23, 2015, doi: 10.1016/j.procir.2015.07.044.

[6] Y. Tokuyama, R. P. C. J. Rajapakse, S. Yamabe, K. Konno, and Y. P. Hung, "A kinect-based augmented reality game for lower limb exercise," Proc. - 2019 Int. Conf. Cyberworlds, CW 2019, pp. 399-402, 2019, doi: 10.1109/CW.2019.00077.

[7] Di. Chatzopoulos, C. Bermejo, Z. Huang, and P. Hui, "Mobile Augmented Reality Survey: From Where We Are to Where We Go," IEEE Access, vol. 5, pp. 6917-6950, 2017, doi: 10.1109/ACCESS.2017.2698164.

[8] H. Peng, "Application Research of AR Holographic Technology based on Natural Interaction in National Culture," Proc. 2019 IEEE 4th Adv Inf. Technol. Electron. Autom. Control Conf. IAEAC 2019, no. Iaeac, pp. 2220-2224, 2019, doi: 10.1109/IAEAC47372.2019.8997672.

[9] S. M. C. Loureiro, J. Guerreiro, and F. Ali, "20 years of research on virtual reality and augmented reality in tourism context: A textmining approach," Tour. Manag., vol. 77, no. October 2019, 2020, doi: 10.1016/j.tourman.2019.104028.

[10] A. Annafi, D. L. Hakim, and D. Rohendi, "Impact of using augmented reality applications in the educational environment," J. Phys. Conf. Ser., vol. 1375 , no. 1 , 2019, doi: 10.1088/1742-6596/1375/1/012080.

[11] A. Moore et al., "Comparative usability of an augmented reality sandtable and 3D GIS for education," Int. J. Geogr. Inf. Sci., vol. 34, no. 2, pp. 229-250, 2020, doi: 10.1080/13658816.2019.1656810.

[12] P. D. Petrov and T. V. Atanasova, "The Effect of augmented reality on students' learning performance in stem education," Inf., vol. 11, no. 4, 2020, doi: 10.3390/INFO11040209.

[13] S. Cai, X. Wang, and F. K. Chiang, "A case study of Augmented Reality simulation system application in a chemistry course," Comput. Human Behav., vol. 37, pp. 31-40, 2014, doi: 10.1016/j.chb.2014.04.018.

[14] M. Kesim and Y. Ozarslan, "Augmented Reality in Education: Current Technologies and the Potential for Education," Procedia - Soc. Behav. Sci., vol. 47, no. 222, pp. 297-302, 2012, doi: 10.1016/j.sbspro.2012.06.654.

[15] J. Porozovs and S. Kristapsone, "The Opinion of Latvian Teachers About the Most Suitable Teaching Methods and Possibilities to Make
Lessons Interesting," J. Pedagog. Psychol. "Signum Temporis," vol. 9, no. 1, pp. 50-56, 2019, doi: 10.1515/sigtem-2017-0009.

[16] N. Pellas, P. Fotaris, I. Kazanidis, and D. Wells, "Augmenting the learning experience in primary and secondary school education: a systematic review of recent trends in augmented reality game-based learning," Virtual Real., vol. 23, no. 4, pp. 329-346, 2019, doi: 10.1007/s10055018-0347-2.

[17] P. Fraga-Lamas, T. M. Fernández-Caramés, Ó. Blanco-Novoa, and M. A. Vilar-Montesinos, "A Review on Industrial Augmented Reality Systems for the Industry 4.0 Shipyard," IEEE Access, vol. 6, pp. 13358-13375, 2018, doi: 10.1109/ACCESS.2018.2808326.

[18] S. R. Dehkordi, M. Ismail, and N. M. Diah, "A review of kinect computing research in education and rehabilitation," Int. J. Eng. Technol., vol. 7, no. 3, pp. 19-23, 2018, doi: 10.14419/ijet.v7i3.15.17399.

[19] F. Garzotto, M. Gelsomini, M. Gianotti, and F. Riccardi, "Engaging children with neurodevelopmental disorder through multisensory interactive experiences in a smart space," Internet of Things, vol. 0, pp. 167-184, 2019, doi: 10.1007/978-3-319-94659-7_9.

[20] M. S. D. R. Guerra and J. Martin-Gutierrez, "Evaluation of fullbody gestures performed by individuals with down syndrome: Proposal for designing user interfaces for all based on kinect sensor," Sensors (Switzerland), vol. 20, no. 14, pp. 1-22, 2020, doi: 10.3390/s20143930.

[21] N. J. Sung, J. Ma, Y. J. Choi, and M. Hong, "Real-time augmented reality physics simulator for education," Appl. Sci., vol. 9, no. 19, pp. 1-12, 2019, doi: 10.3390/app9194019.

[22] T. Matuszka, F. Czuczor, and Z. Sóstai, "Heromirror interactive: A gesture controlled augmented reality gaming experience," ACM SIGGRAPH 2019 Posters, SIGGRAPH 2019, pp. 2-3, 2019, doi: $10.1145 / 3306214.3338554$.

[23] R. Lozada-Yánez, N. La-Serna-Palomino, and F. Molina-Granja, "Augmented Reality and MS-Kinect in the Learning of Basic Mathematics: KARMLS Case," Int. Educ. Stud., vol. 12, no. 9, p. 54, 2019, doi: 10.5539/ies.v12n9p54.

[24] C. Pittman and J. J. L. V. Jr, "PhyAR: Determining the Utility of Augmented Reality for Physics Education in the Classroom," Proc. 2020 IEEE Conf. Virtual Real. 3D User Interfaces, VRW 2020, pp. 761-762, 2020, doi: 10.1109/VRW50115.2020.00231.

[25] M. Zhang, Z. Zhang, Y. Chang, E. S. Aziz, S. Esche, and C. Chassapis, "Recent developments in game-based virtual reality educational laboratories using the microsoft kinect," Int. J. Emerg. Technol. Learn., vol. 13, no. 1, pp. 138-159, 2018, doi: 10.3991/ijet.v13i01.7773.

[26] D. Alexandrovsky, S. Putze, T. Stabbert, T. DÖring, T. FrÖhlich, and R. Malaka, "Demonstrating Vrbox-a virtual reality augmented sandbox," Conf. Hum. Factors Comput. Syst. - Proc., pp. 1-4, 2019, doi: $10.1145 / 3290607.3313251$.

[27] M. Xu et al., "Personalized training through Kinect-based games for physical education," J. Vis. Commun. Image Represent., vol. 62, pp. 394-401, 2019, doi: 10.1016/j.jvcir.2019.05.007.

[28] Y. H. Chang, P. R. Lin, and Y. Te Lu, "Development of a kinect-based english learning system based on integrating the ARCS model with situated learning," Sustain., vol. 12, no. 5, 2020, doi: 10.3390/su12052037.

[29] M. C. Costa, A. Manso, and J. Patrício, "Design of a mobile augmented reality platform with game-based learning purposes," Inf., vol. 11, no. 3, pp. 1-20, 2020, doi: 10.3390/info11030127.

[30] K. E. Uhm et al., "Usefulness of Kinect sensor-based reachable workspace system for assessing upper extremity dysfunction in breast cancer patients," Support. Care Cancer, vol. 28, no. 2, pp. 779-786, 2020, doi: 10.1007/s00520-019-04874-2.

[31] A. Anwer, S. S. Azhar Ali, A. Khan, and F. Meriaudeau, "Underwater 3-D Scene Reconstruction Using Kinect v2 Based on Physical Models for Refraction and Time of Flight Correction," IEEE Access, vol. 5, pp. 15960-15970, 2017, doi: 10.1109/ACCESS.2017.2733003.

[32] Aaboud M, Aad G, Abbott B, Abdinov O, Abeloos B, Abhayasinghe DK, et al. ATLAS Collaboration. Nucl Phys A. 2019;982:985-1009.

[33] "Particle production in proton-proton collisions M. T. Ghoneim," pp. $1-13$.

[34] B. Horn, "The Higgs Field and Early Universe Cosmology: A (Brief) Review," Physics (College. Park. Md)., vol. 2, no. 3, pp. 503-520, 2020, doi: 10.3390/physics2030028. 
[35] Higgs field - Simple English Wikipedia, the free encyclopedia. https://simple.wikipedia.org/wiki/Higgs-field\#cite-ref-eb64-1-0. Accessed 4 Sep 2020

[36] G. Aad et al., "Observation of a new particle in the search for the Standard Model Higgs boson with the ATLAS detector at the LHC," Phys. Lett. Sect. B Nucl. Elem. Part. High-Energy Phys., vol. 716, no. 1, pp. 1-29, 2012, doi: 10.1016/j.physletb.2012.08.020.

[37] "A new boson with a mass of $125 \mathrm{GeV}$ observed with the CMS experiment at the Large Hadron Collider (Science (2012) (1569))," Science, vol. 339, no. 6125. p. 1275, 2013, doi: 10.1126/science.339.6125.1275- b.

[38] P. Azzi, "First look at the physics case of TLEP," Nuovo Cimento della Societa Italiana di Fisica C, vol. 37, no. 2. pp. 11-18, 2014, doi: 10.1393/ncc/i2014-11730-6.

[39] "Broken Symmetries and the Masses of Gauge Bosons - PhysRevLett.13.508." [Online]. Available: http://journals.aps.org/prl/pdf/10.1103/PhysRevLett.13.508.

[40] F. Englert and R. Brout, "Broken symmetry and the mass of gauge vector mesons," Phys. Rev. Lett., vol. 13, no. 9, pp. 321-323, 1964, doi: 10.1103/PhysRevLett.13.321. 